\title{
Micro BIM Adoption: a Multi-Variable Analysis of Adoption within the UK Architecture Sector
}

\author{
Mohamad Kassem ${ }^{\mathrm{a}}$, Ahmed Louay Ahmed ${ }^{\mathrm{b}, \mathrm{c}}$ \\ ${ }^{a}$ Department of Mechanical and Construction Engineering, Northumbria, University Newcastle, United Kingdom \\ ${ }^{b}$ PhD Researcher, Sheffield School of Architecture, University of Sheffield, Sheffield, UK \\ ${ }^{c}$ Lecturer, Department. of Architecture, University of Technology, Baghdad, Iraq
}

\begin{abstract}
To date a comprehensive analysis of interactions among BIM adoption factors, stages of the BIM adoption process, and time (e.g. time intervals of a national BIM initiative) is still lacking. This research aims to profile BIM adoption by mapping such interactions. The analysis is performed for the UK Architecture sector, which was represented with a sample of 177 organisations. To achieve the profiling of BIM adoption in the UK architecture sector, the study uses the outcomes from two types of inferential analysis: an ordinal logistic regression test to identify the 11 top factors influencing the BIM adoption process; and correlation analysis among the 11 top factors at different stages of the BIM adoption process (i.e. awareness, intention, and decision) and across three time intervals (i.e. pre-2011 as the time interval preceding the announcement of the UK BIM mandate, 2011-2016 as the time interval for the implementation of the UK national BIM strategy, and post-2016 as the time interval within which the mandate entered into effect). Capturing the interactions involved in the profiling of micro BIM adoption and assimilating the outcomes in a single model unravel a further understanding of the BIM adoption process. In particular, the results reveal a dynamic behaviour characterising the micro BIM adoption process where: (1) correlated pairs of adoption factors have a varying level of influence within each adoption stage; (2) the factors involved in each pair generally change across the two dimensions (stages of adoption, and time horizon); and (4) the pairs of factors influencing adoption stages over time often combine constructs from the three clusters of drivers identified in [1] (i.e. Innovation/BIM Characteristics, External Environment Characteristics, and Organisation's Internal Environment Characteristics).
\end{abstract}

(C) 2019 The Authors. Published by Budapest University of Technology and Economics \& Diamond Congress Ltd.

Peer-review under responsibility of the scientific committee of the Creative Construction Conference 2019.

Keywords: BIM; BIM Adoption Process; Micro BIM Adoption.

\section{Introduction}

Existing studies on BIM adoption in organisations (i.e., macro BIM adoption) have not significantly addressed the multi-staged nature of the adoption process and to date, our understanding of the BIM adoption process in organisations remain limited. In particular, there is a need to investigate how adoption factors, individually and jointly, contribute to process that culminate in decision to adopt BIM by organisations. This understanding is important for decision makers when developing and implementing micro BIM adoption plans. Existing studies have also used key concepts such as 'implementation', 'adoption' (either as a decision point/milestone, or a process), and 'diffusion' interchangeably [2], [3]. Potential interplays between micro BIM adoption and some market-wide coercive pressures (e.g. Government BIM mandates) over time (e.g. key time intervals of a national BIM initiative) have not been studied in detail [4]. This

*Corresponding authors: mohamad.kassem@northumbria.ac.uk; alaahmed1@ sheffield.ac.uk;

90006@uotechnology.edu.iq 
Mohamad Kassem \& Ahmed Louay Ahmed/ Proceedings of the Creative Construction Conference (2019) 120 https://doi.org/10.3311/CCC2019-120

paper aims to investigate micro BIM adoption by considering these aforementioned interactions. In so doing, the paper will provide a profiling of micro BIM adoption of the UK Architecture sector.

\section{Research methods and findings}

The study is focussed on the first three stages of the BIM adoption process (i.e., awareness, intention, and decision) proposed by Rogers [5]. The sample of organisations involved have all adopted BIM and confirmed its use hence, this is a retrospective study. The study considers the three time intervals that underpin the UK Government BIM national initiative (i.e., pre-announcement of BIM mandate/pre-2011; trial implementation period of BIM mandate/2011-2016; and post-mandate/post-2011). To perform the profiling of BIM adoption within the UK architecture sector, the study used the following steps of data collection and data analysis:

\subsection{Data collection}

To attain the required data for the model, a questionnaire-based survey was sent to 509 architecture practices. 177 valid responses were returned, and 6 incomplete responses were discarded resulting in response rate of $36 \%$. The targeted organisations were listed within the Royal Institute of British Architects (RIBA) as organisations offering BIM services. Participants (i.e. one from each organisation) were selected only if they are either knowledgeable of or were directly involved in the process of BIM adoption within their practice. The questionnaire included two sections: the first section aimed to collect demographic information (i.e. organisation size, number of BIM projects, and dates/timing of BIM adoption decision); the second section included 77 statements covering the 17 constructs. These constructs represent adoption factors organised under the three drivers (i.e., BIM Innovation characteristics; External Environment characteristics, and Internal Environment characteristics) of the Unified BIM Adoption Taxonomy published in Ahmed and Kassem [1]. A five-point Likert scale - ranging from 'strongly disagree' to 'strongly agree'was used to measure the respondents' level of agreement with the various statements representing the measurement items/constructs as explained in the next section .

\subsection{Identifying the most influencing factors on BIM adoption process}

51 (i.e., 17 constructs $\mathrm{x} 3$ adoption stages) hypotheses are formulated by postulating relationship effects between each of 17 factors of the driver clusters (i.e. external environment characteristics, innovation characteristics, and internal environment characteristics) and the three adoption stages (i.e., awareness, intention, and decision). Ordinal Logistic Regression analysis is employed to test the hypotheses. Not only this will help to identify the influencing factors but it will also help ranking these factors according to their influence at each stage of the BIM adoption process. The level of significance of each influencing factor is measured by comparing the P-value for the term (i.e., factor/construct) to the significance level of the null hypothesis (i.e. no association between the term and the response). The significance threshold (denoted as $\alpha$ or alpha) is 0.05 maximum, leaving a 5\% risk of concluding that an association exists when there is not an actual association [6]. The key findings from this stage include:

- The 'Awareness' stage is influenced by six factors associated with the organisational internal environment characteristics and the BIM innovation characteristics. The six factors are: Willingness, Communication behaviour, Observability, Relative advantage, Compatibility, and Social motivations. No significant influence was detectable from the external environment on awareness.

- The 'Intention' stage is affected by nine factors (i.e., Communication behaviour, Relative advantage, Observability, Top management support, Compatibility, Organisation size, Organisational culture, Organisational readiness, and Coercive pressures) from across the three driver clusters including coercive pressure as one of the external environment drivers. 
Mohamad Kassem \& Ahmed Louay Ahmed/ Proceedings of the Creative Construction Conference (2019) 120 https://doi.org/10.3311/CCC2019-120

- The 'Decision' stage is influenced by seven factors (i.e., Communication behaviour, Organisation size, Relative advantage, Compatibility, Coercive pressures, Organisational readiness and Top management support) from across the three driver clusters.

The 11 factors, identified in this step as the most influencing factors on the BIM adoption process, will be used to perform correlation analysis in the next step.

\subsection{Correlation among the most influencing factors}

This section identifies the correlations between the factors affecting each stage of the BIM adoption process while considering the three time intervals (i.e., awareness, intention, and decision) and three time intervals (i.e., preannouncement of BIM mandate/pre-2011; trial implementation period of BIM mandate/2011-2016; and postmandate/post-2011). This analysis resulted in a set of 31 pairs of strong relationships (28 strong positive relationships and 3 negative ones) that are statistically significant (Hiba! A hivatkozási forrás nem található.).

Table 1 The correlations among the 11 most influencing factors (31 pairs of strong relationships) on the BIM adoption process stages (time-dependent)

\begin{tabular}{|c|c|c|c|}
\hline Stage & Time horizon & Pair of correlated factors & Correlation value \\
\hline \multirow[t]{10}{*}{ Awareness } & \multirow[t]{6}{*}{ Pre-2011 } & Observability $\Leftrightarrow$ Relative advantage & $(\mathrm{rs}=.344, \mathrm{p}=.000)$ \\
\hline & & Communication behaviour $\Leftrightarrow$ Compatibility & $(\mathrm{rs}=.341, \mathrm{p}=.000)$ \\
\hline & & Social motivations $\Leftrightarrow$ Willingness & $(\mathrm{rs}=.261, \mathrm{p}=.007)$ \\
\hline & & Communication behaviour $\Leftrightarrow$ Observability & $(\mathrm{rs}=.246, \mathrm{p}=.011)$ \\
\hline & & Relative advantage $\Leftrightarrow$ Social motivations & $(\mathrm{rs}=.212, \mathrm{p}=.029)$ \\
\hline & & Observability $\Leftrightarrow$ Compatibility & $(\mathrm{rs}=.210, \mathrm{p}=.031)$ \\
\hline & \multirow[t]{3}{*}{$2011-2016$} & Observability $\Leftrightarrow$ Relative advantage & $(\mathrm{rs}=.272, \mathrm{p}=.024)$ \\
\hline & & Relative advantage $\Leftrightarrow$ Social motivations & $(\mathrm{rs}=.263, \mathrm{p}=.029)$ \\
\hline & & Social motivations $\Leftrightarrow$ Willingness & $(\mathrm{rs}=.255, \mathrm{p}=.035)$ \\
\hline & Post-2016 & Excluded /inadequate statistically & N/A \\
\hline \multirow[t]{11}{*}{ Intention } & \multirow[t]{5}{*}{ Pre-2011 } & Organisational culture $\Leftrightarrow$ Relative advantage & $(\mathrm{rs}=.491, \mathrm{p}=.001)$ \\
\hline & & Observability $\Leftrightarrow$ Top management support & $(\mathrm{rs}=.373, \mathrm{p}=.013)$ \\
\hline & & Communication behaviour $\Leftrightarrow$ Organisational readiness & $(\mathrm{rs}=.336, \mathrm{p}=.026)$ \\
\hline & & Top management support $\Leftrightarrow$ Coercive pressures & $(\mathrm{rs}=.334, \mathrm{p}=.027)$ \\
\hline & & Communication behaviour $\Leftrightarrow$ Compatibility & $(\mathrm{rs}=.300, \mathrm{p}=.047)$ \\
\hline & \multirow[t]{5}{*}{$2011-2016$} & Observability $\Leftrightarrow$ Relative advantage & $(\mathrm{rs}=.386, \mathrm{p}=.000)$ \\
\hline & & Communication behaviour $\Leftrightarrow$ Observability & $(\mathrm{rs}=.286, \mathrm{p}=.001)$ \\
\hline & & Organisational culture $\Leftrightarrow$ Relative advantage & $(\mathrm{rs}=.274, \mathrm{p}=.002)$ \\
\hline & & Communication behaviour $\Leftrightarrow$ Compatibility & $(\mathrm{rs}=.211, \mathrm{p}=.019)$ \\
\hline & & Relative advantage $\Leftrightarrow$ Organisational readiness & $(\mathrm{rs}=.192, \mathrm{p}=.034)$ \\
\hline & Post-2016 & Compatibility $\Leftrightarrow$ Coercive pressures & $(\mathrm{rs}=.911, \mathrm{p}=.000)$ \\
\hline \multirow[t]{11}{*}{ Decision } & \multirow[t]{3}{*}{ Pre-2011 } & Communication behaviour $\Leftrightarrow$ Relative advantage & $(\mathrm{rs}=-.424, \mathrm{p}=.017)$ \\
\hline & & Coercive pressures $\Leftrightarrow$ Top management support & $(\mathrm{rs}=.375, \mathrm{p}=.038)$ \\
\hline & & Communication behaviour $\Leftrightarrow$ Organisational readiness & $(\mathrm{rs}=.367, \mathrm{p}=.043)$ \\
\hline & \multirow[t]{4}{*}{$2011-2016$} & Communication behaviour $\Leftrightarrow$ Organisation size & $(\mathrm{rs}=-.189, \mathrm{p}=.049)$ \\
\hline & & Communication behaviour $\Leftrightarrow$ Compatibility & $(\mathrm{rs}=.302, \mathrm{p}=.001)$ \\
\hline & & Communication behaviour $\Leftrightarrow$ Relative advantage & $(\mathrm{rs}=.243, \mathrm{p}=.011)$ \\
\hline & & Relative advantage $\Leftrightarrow$ Organisational readiness & $(\mathrm{rs}=.210, \mathrm{p}=.029)$ \\
\hline & \multirow[t]{4}{*}{ Post-2016 } & Relative advantage $\Leftrightarrow$ Organisational readiness & $(\mathrm{rs}=.414, \mathrm{p}=.011)$ \\
\hline & & Compatibility $\Leftrightarrow$ Top management support & $(\mathrm{rs}=.355, \mathrm{p}=.031)$ \\
\hline & & Relative advantage $\Leftrightarrow$ Compatibility & $(\mathrm{rs}=.348, \mathrm{p}=.035)$ \\
\hline & & Coercive pressures $\Leftrightarrow$ Organisational readiness & $(\mathrm{rs}=-.352, \mathrm{p}=.033)$ \\
\hline
\end{tabular}

These results are visualised in Figure 1 using a two-dimensional model, with one dimension being the stages of the BIM adoption process and the other dimension being the time-horizon, with the following conventions:

- The pairs of factors influencing awareness were top-to-bottom (A to F) arranged according to their actual power of influence. For example, in Hiba! A hivatkozási forrás nem található. the first block of the 'Awareness stage' in 
Mohamad Kassem \& Ahmed Louay Ahmed/ Proceedings of the Creative Construction Conference (2019) 120 https://doi.org/10.3311/CCC2019-120

the Pre-2011 period ranks the following pairs from top-to-bottom in a descending order: [i.e. Observability $\Leftrightarrow$ Relative advantage ( $\mathrm{rs}=.344, \mathrm{p}=.000$ ); Communication behaviour $\Leftrightarrow$ Compatibility ( $\mathrm{rs}=.341, \mathrm{p}=.000$ ); Social motivations $\Leftrightarrow$ Willingness ( $\mathrm{rs}=.261, \mathrm{p}=.007$ ); Communication behaviour $\Leftrightarrow$ Observability ( $\mathrm{rs}=.246, \mathrm{p}=.011$ ); Social motivations $\Leftrightarrow$ Relative advantage ( $\mathrm{rs}=.212, \mathrm{p}=.029$ ); and Observability $\Leftrightarrow$ Compatibility ( $\mathrm{rs}=.210$, $\mathrm{p}=.031)]$.

- The level of influence of each pair is symbolised using simple pie diagrams: 1 full circle for $\mathrm{p}$-values between $\mathrm{p}=0.000-\mathrm{p}=0.009 ; 3 / 4$ circle for $\mathrm{p}$-values between $\mathrm{p}=0.01-\mathrm{p}=0.025 ; 1 / 2$ circle for $\mathrm{p}$-values between $\mathrm{p}=0.026-$ $\mathrm{p}=0.04$; and $1 / 4$ circle for $\mathrm{p}$-values between $\mathrm{p}=0.041-\mathrm{p}=0.05$.

Three colour codes are used to identify the factors appurtenance to the three driver clusters. Pink, orange, and blue are used for the External Environment Characteristics, Innovation/BIM Characteristics, and Organisation's Internal Environment Characteristics, respectively.

The profiling of micro BIM adoption with the model (Figure 1) presents an integrated view of the adoption problem by addressing the interactions between: pairs of correlated adoption factors, stages of the BIM adoption process, and the time horizon. The model shows that micro BIM adoption is characterised by a dynamic behaviour where:

- Correlated pairs of factors exert a varying level of influence within each adoption stage and across different stages and time horizons. For example, the pair "Communication behaviour $\Leftrightarrow$ Compatibility" has a continuous influence with its effect transferring (a) across three consecutive stages within two periodical time-horizon (i.e., from 'Awareness' to 'Intention' in Pre-2011, then from 'Intention' to 'Decision' in 2011-2016), and (b) across two consecutive periodical time-horizon of the same stage (i.e., from Pre-2011 to 2011-2016 at 'Intention'). Hence, this pair plays a role in driving the organisation to: gain knowledge about the innovation (i.e. formulate awareness); develop a favourable attitude (i.e., Intention) towards BIM adoption; and move towards formulating the decision to adopt BIM;

- Factors involved in each correlated pair influence BIM adoption generally change across the adoption stages and the time horizon. For example, the pairs (Relative advantage $\Leftrightarrow$ Observability), (Communication behaviour $\Leftrightarrow$ Compatibility), and (Relative advantage $\Leftrightarrow$ Organisational readiness) all change the composition of the pair involved in exerting influence on the BIM adoption process; and

- Pairs of factors influencing adoption stages over time often combine constructs from the three clusters of drivers (i.e. Innovation/BIM Characteristics, External Environment Characteristics, and Organisation's Internal Environment Characteristics). This indicates that micro BIM adoption is a dynamics system whose understanding require the simultaneous contemplation of these three environments.

These findings, by revealing the different types of interactions between the factors affecting the BIM adoption process across two important dimensions (i.e. stages of the BIM adoption process stages, and time) improve the understanding of the micro BIM adoption. And by so doing, it could inform micro BIM adoption implementation plans. For example, in the case of Pair B (i.e. communication behaviour and compatibility) influencing the awareness stage at pre-2011, a decision maker may implement activities that change the communication behaviour (i.e. the degree of openness and engagement of an organisation with social groupings and networks interested in innovation adoption and promotion) of an organisation to make the organisation perceives the innovation as more compatible with their previous experiences and current needs and values. Further, in organisations where readiness was achieved between [2011 2016] other pairs of correlated factors are involved, and none of these pairs entails factors from Pair B (communication behaviour and compatibility). By interpreting the model's results in this way, decision makers may be able to calibrate and tailor micro BIM adoption activities to their special circumstances depending on their position across both the adoption and time dimensions. 
Mohamad Kassem \& Ahmed Louay Ahmed/ Proceedings of the Creative Construction Conference (2019) 120 https://doi.org/10.3311/CCC2019-120

\section{Conclusion}

This paper performed the profiling of micro BIM adoption within the UK architecture sector. The profiling of micro BIM adoption captured interactions between adoption factors, BIM adoption stages, and time. The time dimension was split into three time intervals denoting key phases of the UK national BIM initiative. The profiling of BIM adoption was achieved from two different sets of results: (1) 11 top influencing factors with positive and significant influence on the BIM adoption stages identified from the Ordinal Logistic Regression;; and (2) correlation analysis among the 11 factors at different stages of the BIM adoption process and at different time intervals, which resulted in a set of 31 pairs of strong relationships ( 28 strong positive relationships and 3 negative ones). These two set of results were used to build a model which provided an integrated view of micro BIM adoption by capturing the interactions between pairs of correlated factors, the BIM adoption process, and time. The model was used to show some of the dynamic patterns inherent in micro BIM adoption. The model introduced a new 'dynamic' view of micro BIM adoption where the potential influence, the level of the influence, and the factors exerting the influence vary according to both the stage of the BIM adoption process, and time. In future, the work will be extended to include organisation size (i.e. micro, small, medium, and large) as an additional variable involved in this dynamic view of micro BIM adoption.

\section{Acknowledgements}

This work has been sponsored by the Iraqi Ministry of Higher Education and Scientific Research (MOHESR) jointly with the University of Technology, Baghdad, Iraq.

\section{References}

[1] Ahmed, A.L. and M. Kassem, A unified BIM adoption taxonomy: Conceptual development, empirical validation and application. Automation in Construction, 2018. 96: p. 103-127. https://doi.org/10.1016/j.autcon.2018.08.017

[2] Al-Shammari, M.A. An appraisal of the protocol that was published by the Construction Industry Council (CIC) to facilitate the use of Building Information Modelling (BIM) on projects. in 30th Annual Association of Researchers in Construction Management Conference, ARCOM 2014. 2014. Association of Researchers in Construction Management.

[3] Haron, A.T., et al., Building information modelling:A case study approach to identify readiness criteria for process requirement. AmericanEurasian Journal of Sustainable Agriculture, 2014. 8(7 SPEC. ISSUE 2): p. 85-91.

[4] Hosseini, M.R., et al., BIM adoption within Australian Small and Medium-sized Enterprises (SMEs): an innovation diffusion model. Construction Economics and Building, 2016. 16(3): p. 71-86. https://doi.org/10.5130/AJCEB.v16i3.5159

[5] Rogers, E.M., Diffusion of Innovations, 5th Edition. 2003: Free Press.

[6] Harrell, F.E., Ordinal logistic regression, in Regression modeling strategies. 2001, Springer. p. 331-343. 
Mohamad Kassem \& Ahmed Louay Ahmed/ Proceedings of the Creative Construction Conference (2019) 120 https://doi.org/10.3311/CCC2019-120

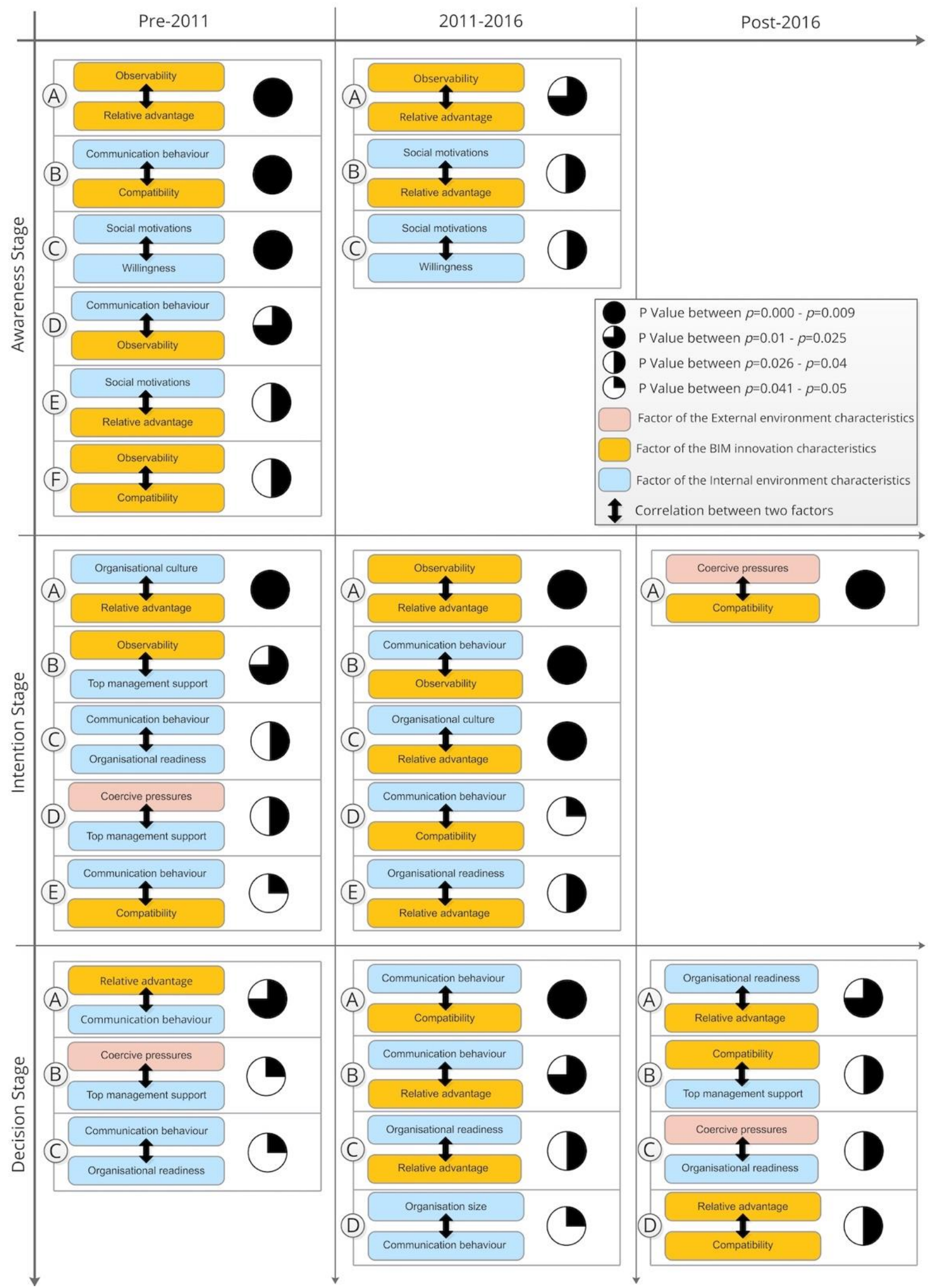

Figure 1 A two-dimensional model profiling BIM adoption process in UK Architecture Sector 